\title{
Individual Differences in Stressor-Evoked Blood Pressure Reactivity Vary with Activation, Volume, and Functional Connectivity of the Amygdala
}

\author{
Peter J. Gianaros, ${ }^{1,2}$ Lei K. Sheu, ${ }^{1}$ Karen A. Matthews,,${ }^{1,2,3}$ J. Richard Jennings, ${ }^{1,2}$ Stephen B. Manuck, ${ }^{2}$ and \\ Ahmad R. Hariri ${ }^{1,2}$ \\ Departments of ${ }^{\text {Psychiatry, }}{ }^{2}$ Psychology, and ${ }^{3}$ Epidemiology, University of Pittsburgh, Pittsburgh, Pennsylvania 15213
}

\begin{abstract}
Individuals who exhibit exaggerated blood pressure reactions to psychological stressors are at risk for hypertension, ventricular hypertrophy, and premature atherosclerosis; however, the neural systems mediating exaggerated blood pressure reactivity and associated cardiovascular risk in humans remain poorly defined. Animal models indicate that the amygdala orchestrates stressor-evoked blood pressure reactions via reciprocal signaling with corticolimbic and brainstem cardiovascular-regulatory circuits. Based on these models, we used a multimodal neuroimaging approach to determine whether human individual differences in stressor-evoked blood pressure reactivity vary with amygdala activation, gray matter volume, and functional connectivity with corticolimbic and brainstem areas implicated in stressor processing and cardiovascular regulation. We monitored mean arterial pressure (MAP) and concurrent functional magnetic resonance imaging BOLD signal changes in healthy young individuals while they completed a Stroop color-word stressor task, validated previously in epidemiological studies of cardiovascular risk. Individuals exhibiting greater stressor-evoked MAP reactivity showed (1) greater amygdala activation, (2) lower amygdala gray matter volume, and (3) stronger positive functional connectivity between the amygdala and perigenual anterior cingulate cortex and brainstem pons. Individual differences in amygdala activation, gray matter volume, and functional connectivity with corticolimbic and brainstem circuits may partly underpin cardiovascular disease risk by impacting stressor-evoked blood pressure reactivity.
\end{abstract}

Key words: amygdala; blood pressure reactivity; cardiovascular disease risk; functional connectivity; individual differences; gray matter volume; stress

\section{Introduction}

A rise in blood pressure is a hallmark of the prototypical cardiovascular reaction to an acute psychological stressor. As the net product of changes in cardiac output and regional vascular resistance that shunt blood away from the viscera and toward large muscle groups, stressor-evoked blood pressure reactions provide hemodynamic support for adaptive behavioral action (Cannon, 1928). Some individuals, however, exhibit large-magnitude or so-called "exaggerated" stressor-evoked blood pressure reactions that may contribute to pathophysiological changes in the cardiovascular system, particularly when expressed recurrently over the lifespan (Krantz and Manuck, 1984). Such changes may include myocardial and vascular remodeling, vascular shear stress, and endothelial cell injury (Treiber et al., 2003). Epidemiological evidence further indicates that individuals who exhibit exaggerated stressor-evoked blood pressure reactions are at risk for hyperten-

Received Aug. 8, 2007; revised Nov. 18, 2007; accepted Dec. 7, 2007.

This work was supported by National Institutes of Health Grants K01 MH070616 (P.J.G.) and HL 076852/076858 (K.A.M.). Drs. Gary G. Berntson and Israel C. Christie and two anonymous reviewers provided constructive comments on a draft of this manuscript.

Correspondence should be addressed to Peter J. Gianaros, Department of Psychiatry, University of Pittsburgh, 3811 0'Hara Street, Pittsburgh, PA 15213. E-mail: gianarospj@upmc.edu.

DOI:10.1523/JNEUROSCI.3606-07.2008

Copyright $\odot 2008$ Society for Neuroscience $\quad$ 0270-6474/08/280990-10\$15.00/0 sion, ventricular hypertrophy, and premature atherosclerosis in major arteries (Treiber et al., 2003; Jennings et al., 2004; Matthews et al., 2006b). Despite this epidemiological evidence, little is known about the neural systems that link the central processing of stressors to human individual differences in blood pressure reactivity and associated cardiovascular risk.

Cumulative evidence from animal models, however, indicates that the amygdala plays an instrumental role in linking stressor processing with brainstem autonomic-cardiovascular control mechanisms that regulate blood pressure. Lesion, early gene expression, and electrical and chemical stimulation studies specifically show that the visceromotor output area of the amygdala, the central nucleus, directly coordinates changes in blood pressure with stress-related behaviors via projections to brainstem preautonomic areas (Reis and LeDoux, 1987; Dampney, 1994; Saha, 2005). Noteworthy in the context of cardiovascular risk, central nucleus lesions blunt exaggerated stressor-evoked blood pressure reactions in rats genetically prone to hypertension (Galeno et al., 1984; Sanders et al., 1994) and prevent the development of hypertension induced by chronic stress (Fukumori et al., 2004) and fear conditioning (Baklavadzhyan et al., 2000). Thus, animal evidence suggests that the amygdala could mediate dysregulated stressor-evoked blood pressure reactions, possibly contributing in part to cardiovascular disease vulnerability. 
Accordingly, we tested whether human individual differences in stressor-evoked blood pressure reactivity varied with concurrent levels of amygdala activation in healthy young adults who completed a performance-titrated Stroop color-word stressor task that has been validated in epidemiological studies of cardiovascular risk (Jennings et al., 2004). In extension, we used a computational neuroanatomical method, optimized voxel-based morphometry (Ashburner and Friston, 2000; Good et al., 2001), to explore whether individual differences in amygdala gray matter volume could account for observed associations between amygdala activation and blood pressure reactivity. Furthermore, we tested whether individual differences in blood pressure reactivity varied with the functional connectivity between the amygdala and pons, a brainstem area networked with the amygdala and considered as a key node in the preautonomic circuitry (above the medulla) critical for blood pressure control (Hopkins and Holstege, 1978; Miller et al., 1991; Dampney, 1994). Finally, consistent with evidence that the amygdala is but one component of a distributed corticolimbic circuit involved in processing behaviorally salient stimuli and regulating visceromotor function (LeDoux, 2000; Öngür and Price, 2000; Davis and Whalen, 2001; Sah et al., 2003; Zald, 2003; Seeley et al., 2007), we explored whether stressor-evoked blood pressure reactivity varied with volumetric-, activation-, or connectivity-related changes in corticolimbic areas networked with the amygdala; namely, the cingulate, medial prefrontal, and insular cortices.

\section{Materials and Methods \\ Participants}

Participants were 32 right-handed undergraduates (12 men; mean age, $19.90 \pm 1.30 \mathrm{SD})$. As determined by a medical history interview, participants were free of (1) current or lifetime history of any cardiovascular disease or surgery (including hypertension, stroke, myocardial infarction, congestive heart failure, atrial or ventricular arrhythmias, coronary bypass, carotid artery, or peripheral vascular surgery); (2) current or lifetime history of cancer, a chronic kidney or liver condition, type I or II diabetes, or any pulmonary or respiratory disease; (3) current or lifetime diagnoses of any substance abuse or psychiatric syndrome; (4) current or lifetime use of glucocorticoid medications for $>1$ month; (5) a previous cerebrovascular accident or trauma involving loss of consciousness; (6) previous neurosurgery or history of a neurological condition; (7) pregnancy (females); (8) color blindness; (9) claustrophobia; and (10) metallic implants. Participants were normotensive, as determined by the average of three seated resting blood pressure readings (mean, 115.68/64.35 $\mathrm{mmHg}, \mathrm{SD}, 8.56 / 9.38$ ). For other sample characteristics, see supplemental Table 1 (available at www.jneurosci.org as supplemental material). To minimize extraneous influences on blood pressure, participants abstained from eating, exercising, and consuming caffeinated and tobacco products for $3 \mathrm{~h}$ and drinking alcoholic beverages for $12 \mathrm{~h}$ before testing. The University of Pittsburgh Institutional Review Board granted study approval; each participant gave informed consent and received $\$ 30.00$ (U.S.).

\section{Blood pressure reactivity task}

To evoke blood pressure reactivity, participants completed a modified performance-titrated Stroop color-word interference task (Gianaros et al., 2005). In this task, participants completed six blocks of color-word identification trials comprising two alternating 54-58 s conditions, termed congruent and incongruent conditions (Fig. 1). Before each condition, participants fixated on a crosshair (displayed for a variable 12-16 s period), and then in single trials identified the color in which a target word was shown by selecting (as quickly and accurately as possible) one of four identifier words naming the color of the target. On each trial, a target word was centered in a visual display, and four randomly ordered identifier words appeared in one row at bottom. Participants selected an identifier by pressing one of four corresponding buttons on a response glove. The selected identifier was outlined and the correct identifier was

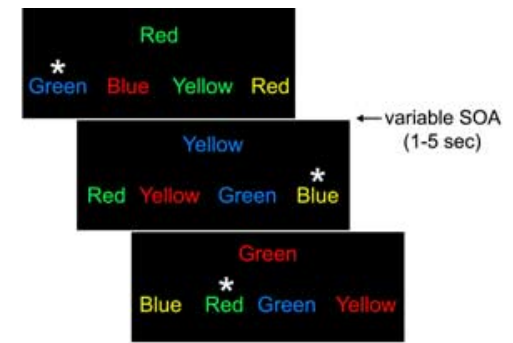

Example Incongruent Condition Trials

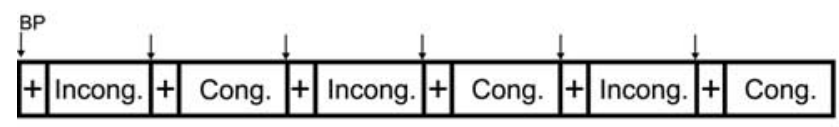

\section{Example Congruent}

Condition Trials

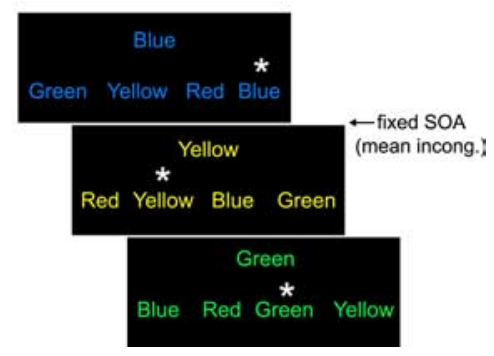

Figure 1. Sample trials from the incongruent (top) and congruent (bottom) conditions of a Stroop task designed to elicit blood pressure reactivity in a blocked fMRI paradigm (center). Participants completed three blocks of each $54-58$ s condition, which were interleaved with a 12-16 s fixation $(+)$ period. The task was to identify the color of the center target on each trial by selecting one of four identifier words at bottom. For the incongruent condition, trials were presented every $1-5 \mathrm{~s}$, with a variable SOA that maintained task accuracy at $\sim 70 \%$ within and between participants (see Materials and Methods). For the congruent condition, trials were presented at a fixed SOA, defined by the mean SOA of the preceding incongruent condition. For illustration, correct responses have an asterisk above the correct identifier. To measure blood pressure (BP), a brachial BP cuff was inflated on the left arm during each fixation period (indicated by an $\downarrow$ in the center schematic); a single mean arterial pressure reading was obtained approximately at the end of each condition.

highlighted before the next trial. For all trials in the congruent condition, (1) the target word appeared in a color congruent with the target word, and (2) all identifiers appeared in the same color as the target. For all trials in the incongruent condition, (1) the target word appeared in a color incongruent with the target word and (2) all of the identifiers appeared in colors incongruent with the colors that the identifier words named.

To elicit blood pressure reactivity while controlling for individual differences in task performance, each participant's accuracy at target word identification was titrated to (and maintained at) $\sim 70 \%$ during each incongruent condition by adjusting trial presentation times in $500 \mathrm{~ms}$ steps within a range of $1-5 \mathrm{~s}$. Specifically, more accurate performance within a given incongruent condition prompted shorter response time windows in which to select an identifier word; conversely, less accurate performance lengthened response windows.

To control for trial frequency and motor response differences between congruent and performance-titrated incongruent conditions, the number of trials administered in each congruent condition was matched to the number of trials completed in the preceding incongruent condition. To implement this control, a block of incongruent trials was administered first, and congruent condition trials were administered at a stimulus onset asynchrony (SOA) determined by the mean SOA of the preceding incongruent block. Consequently, the average number of trials completed during the congruent condition (mean, 16.9; SD, 7.0) approximated the number completed in the incongruent condition (mean, 16.0; SD, 7.0). Participants received task instructions before scanning, but were not informed that performance would be titrated in the incongruent condition. Post hoc, we confirmed that accuracy in the incongruent condition was titrated to $68.46 \%$ (SD, 6.53 ); by comparison, mean accuracy was $92.58 \%(\mathrm{SD}, 5.21)$ in the congruent condition $\left(t_{(31)}=17.60\right.$; $p<0.001$ by $t$ test $)$. 
Assessment of stress and blood pressure

Stress. To assess stress ratings of the task conditions as a manipulation check after imaging, participants completed five-point scales $(0$, not at all stressful; 4, extremely stressful).

Blood pressure. Oscillometric recordings of mean arterial blood pressure (MAP) were taken from the brachial artery of the left arm (not used for task responding) with an automated monitor (model 3155MVS; InVivo Research, Orlando, FL). Resting MAP was recorded every 2 min during an 8 -min baseline during which structural brain images were acquired before the task. MAP was recorded every 1 min during the task, such that cuff inflation coincided with the fixation period initiating each condition (this ensured that the conditions were matched for procedural effects caused by cuff inflation). Resting MAP was computed as the average of the four baseline recordings. Task-related blood pressure was computed as the average of the three recordings taken approximately at the end of each incongruent condition. The task-baseline MAP difference was used as an indicator of MAP reactivity, following the epidemiological definition of cardiovascular reactivity: a change from rest to a period of psychological stress, evoked here by the incongruent condition (Kamarck and Lovallo, 2003).

\section{Acquisition of brain imaging data}

Imaging data were acquired on a 3T Siemens (Munich, Germany) Allegra head-dedicated scanner equipped with a standard radio-frequency head coil. Soft foam padding was used to restrain head movement. For anatomical cross-registration and normalization of $\mathrm{T} 2^{\star}$-weighted BOLD images, coplanar T2-weighted images were acquired before the Stroop task [repetition time (TR), $6440 \mathrm{~ms}$; echo time (TE), $73 \mathrm{~ms}$; flip angle, $150^{\circ}$; 39 axial-oblique slices; field of view (FOV), $200 \mathrm{~mm}$; voxel resolution, $0.8 \times 0.8 \times 3.0 \mathrm{~mm}$; slice thickness, $3 \mathrm{~mm}$ with no gap]. During the task, BOLD images were acquired in one imaging run with a gradientecho echo planar imaging sequence (TR, $2000 \mathrm{~ms}$; TE, $25 \mathrm{~ms}$; flip angle, $79^{\circ}$; 39 axial-oblique slices; FOV, $200 \mathrm{~mm}$; in-plane voxel resolution, $3.125 \times 3.125$ square $\mathrm{mm}$; slice thickness, $3 \mathrm{~mm}$ with no gap; four initial discarded volumes). For optimized voxel-based morphometry (VBM), high-resolution T1-weighted images were obtained after functional imaging [three-dimensional fast-gradient magnetization prepared rapid gradient-echo (MPRAGE) sequence: TR, $1540 \mathrm{~ms}$; TE, $3.04 \mathrm{~ms} ; 192 \mathrm{sag}-$ ittal slices; flip angle, $8^{\circ}$; FOV , $256 \mathrm{~mm}$; slice thickness, $1 \mathrm{~mm}$ with no gap; voxel dimensions, $1 \mathrm{~mm}^{3}$ ].

\section{Preprocessing and analysis of functional, volumetric, and functional connectivity data}

All imaging data were preprocessed and analyzed with statistical parametric mapping software (SPM5; http://www.fil.ion.ucl.ac.uk/spm), operating in the Matlab software environment (version 7.1; MathWorks, Natick, MA).

Functional data. For functional image preprocessing, BOLD images were unwarped and realigned to the first image of the series to correct for spatial displacement. Realigned images were then coregistered to the anatomical space of each participant's T2-weighted structural image. Coregistered images were normalized with a 12-parameter affine model to the anatomical space of the Montreal Neurological Institute (MNI) template. Normalized images were smoothed with an $8 \mathrm{~mm}$ full-width at half-maximum (FWHM) isotropic Gaussian kernel to accommodate individual differences in anatomy and to meet the distributional assumptions of random field theory for statistical testing (Friston et al., 1995, 1996).

After preprocessing, a contrast image of BOLD activation (incongruent $>$ congruent) was estimated for each individual. To generate contrast images, BOLD signal changes for each condition (fixation, congruent, incongruent) were modeled with a rectangular waveform convolved with the default SPM hemodynamic response function. Next, task-related BOLD activation was estimated with a design matrix specifying a general linear model (GLM) that included explanatory regressors for the fixation, congruent, and incongruent epochs [linear BOLD signal drifts were removed with a high-pass filter $(128 \mathrm{~s})$; serial correlations in the BOLD signal were accounted for by first-degree autoregressive correction].

To determine the association between task-related BOLD activation and MAP reactivity across individuals, individual contrasts were submitted to a multiple regression analysis. For the regression, MAP reactivity was entered as an explanatory factor and sex as a covariate. Given the study's primary focus on the amygdala, this area was targeted as a region of interest (ROI) using an anatomical mask created with the Wake Forest University (WFU) Pick Atlas (Maldjian et al., 2003). The mask was dilated by an expanding factor of 1 to fully encompass the amygdala (Etkin et al., 2004). To control for multiple statistical testing within the expanded search volume of the extended bilateral amygdala $\left(11,988 \mathrm{~mm}^{3}\right)$, we maintained a false positive detection rate at $p<0.05$ by using a threshold of $p<0.005$ with a cluster $[k]$ extent of at least 17 contiguous voxels (Forman et al., 1995). The number of contiguous voxels needed to maintain this false positive detection rate was empirically determined by Monte Carlo simulations implemented in AlphaSim, which accounted for spatial correlations between BOLD signal changes in neighboring voxels (Ward, 2000). In addition to ROI analyses of the amygdala, we executed a whole-brain analysis of task-related activation and associated individual differences in MAP reactivity controlling for voxelwise multiple testing with a familywise error rate (FWE) threshold of $p<0.05$ (Friston et al., 1996).

Volumetric data. Regional and total gray matter volumes from T1weighted MPRAGE images were determined using optimized VBM procedures implemented with MATLAB scripts written by J. Ashburner and C. Gaser (available at http://dbm.neuro.uni-jena.de/vbm.html). MPRAGE images were first used to generate a study-specific T1weighted template and tissue-specific templates for gray matter, white matter, and CSF, all normalized to MNI space and smoothed with an 8 mm FWHM spatial filter. Individual MPRAGE images were then segmented into gray, white, and CSF images with a mixture model cluster analysis using study-specific tissue templates as Bayesian prior probability maps of tissue intensity. An additional Hidden Markov Random Field model was used to optimize tissue segmentation (Cuadra et al., 2005), and image intensities were bias-corrected to minimize the influence of magnetic field inhomogeneities. Resulting gray matter images were normalized to the study-specific gray matter template with affine transformations and smooth basis functions that minimized global and nonlinear differences between each image and the template. Subsequently, gray matter voxel values were multiplied by the Jacobian matrix parameters derived from normalization. Consequently, relative volume changes introduced by spatial deformation were incorporated into each voxel. For analysis, volumetric gray matter images were smoothed at $8 \mathrm{~mm}$ FWHM.

To determine associations between MAP reactivity and amygdala gray matter volume, volumetric gray matter images were submitted to a multiple regression analysis. Following the approach for functional data analysis, MAP reactivity was entered as an explanatory factor and sex as a covariate. Total gray matter volume was entered as an additional covariate to account for the influence of individual differences in global gray matter. For the hypothesis-driven ROI analysis of the amygdala, we used the same amygdala mask used for functional analyses, and we maintained a false positive detection rate of $p<0.05$ by applying height and extent thresholds of $p<0.005$ and $k=156$, as determined by Monte Carlo simulation. In a subsequent exploratory whole-brain analysis, we then tested for associations between MAP reactivity and regional gray matter volume controlling for voxelwise multiple testing using height and extent thresholds of $p<0.005$ and $k=750$, as no associations survived wholebrain correction at a more conservative FWE threshold of $p<0.05$.

Connectivity data. In a set of functional connectivity analyses, we first tested whether MAP reactivity varied with temporally coupled BOLD signal changes in the amygdala and pons. Connectivity estimates reported here are taken to reflect functionally relevant correlations between components of neural circuits (Friston, 1994); however, they do not establish the causal or time-lagged nature of regional neural coupling. First, we used MarsBaR (Brett et al., 2002) to extract the mean BOLD time series in the left and right amygdala for each individual. Specifically, each time series was extracted from the mean BOLD signal of all voxels in a $5 \mathrm{~mm}$ radius surrounding the amygdala coordinates identified in the regression analyses of amygdala activation and MAP reactivity described above and reported below (left amygdala $x, y, z$ MNI seed coordinates: $-27,0,-15$; right: $27,3,-12$ ). Second, extracted time 

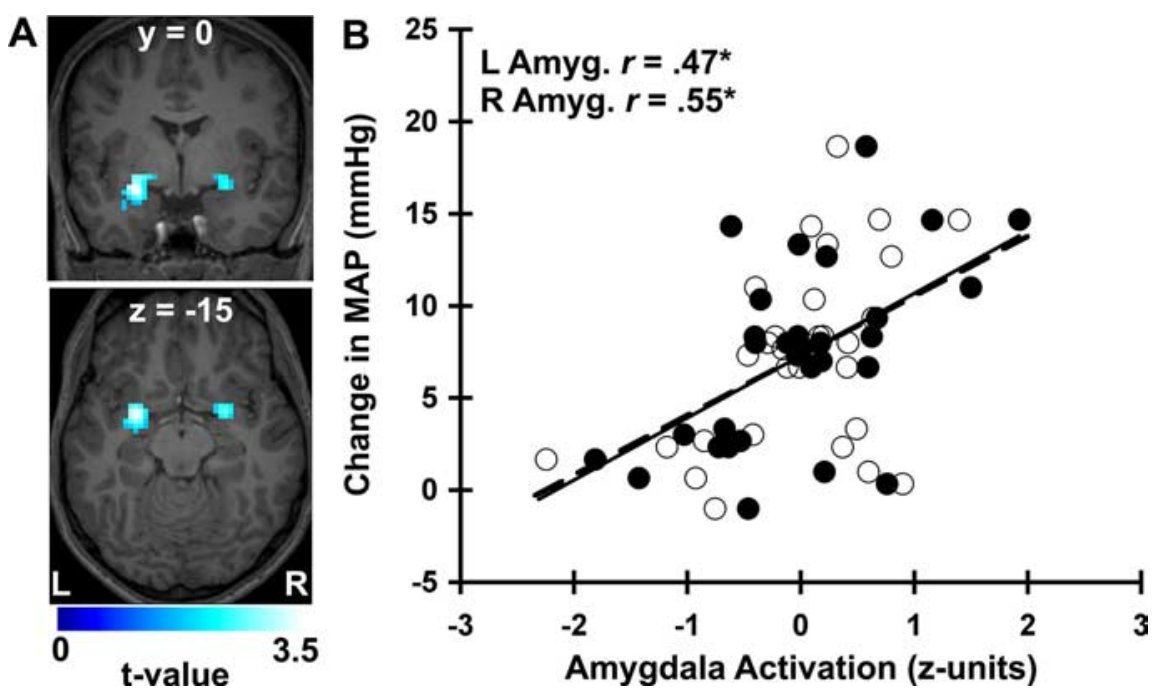

Figure 2. Greater MAP reactivity varied with greater amygdala activation to the incongruent condition. $A$, Statistical parametric maps from an ROI regression analysis identifying clusters of the left $\left(x, y, z\right.$ MNI coordinates: $-27,0,-15 ; t_{(29)}=3.57$, $\left.p_{\text {uncorrected }}=0.001, p_{\mathrm{FWE}}=0.045 ; k=124\right)$ and right $\left(27,3,-12 ; t_{(29)}=2.86, p_{\text {uncorrected }}=0.004, p_{\mathrm{FWE}}=0.14, k=28\right)$ amygdala where MAP reactivity varied with activation after covariate control for sex. Parametric maps are projected onto coronal (top) and axial (bottom) sections of an averaged MNI-transformed T1-weighted template derived from study participants, as in remaining figures. $\boldsymbol{B}$, MAP reactivity (change from a resting baseline) is shown as a function of mean-centered and standardized amygdala BOLD activation values extracted from the peak voxels of the left ( $L$; open circles, dashed line) and right ( $R$; closed circles, solid line) amygdala clusters profiled in $\boldsymbol{A} .{ }^{*} p<0.01$.

series were mean centered and drift corrected. Third, values exceeding 3 $\mathrm{SD}$ of the mean of the drift-corrected time series were replaced by the average of 2 adjacent values. Finally, the corrected time series were entered as regressors in the individual GLM design matrices described above. For each individual, this yielded a functional connectivity map identifying areas where BOLD signal changes were temporally coupled with signal changes derived from the left and right amygdala seeds (cf., Greicius et al., 2003; Seeley et al., 2007).

In a hypothesis-driven ROI regression analysis with covariate control for sex, we tested whether MAP reactivity varied with the functional connectivity between the amygdala and pons using a bilateral mask of the pons generated with the WFU Pick Atlas. To maintain a false positive detection rate of $p<0.05$ within the pons ROI volume $\left(6777 \mathrm{~mm}^{3}\right)$, we applied height and extent thresholds of $p<0.005$ and $k=18$, determined by Monte Carlo simulation. To identify other areas exhibiting functional connectivity with the amygdala during the Stroop task, we executed whole-brain analyses that controlled for voxelwise multiple testing with height and extent thresholds of $p_{\mathrm{FWE}}<0.001$ and $k=15$ (cf., Greicius et al., 2003; Seeley et al., 2007).

\section{Results}

\section{Stress ratings and MAP reactivity}

The performance-titrated incongruent Stroop condition elicited moderate subjective stress sufficient to evoke individual differences in blood pressure reactivity. Specifically, participants rated the incongruent condition as more stressful than the congruent condition on a 0 to 4 scale [mean, $2.66 \pm 0.79 \mathrm{SD}$ vs $0.81 \pm 0.69$; $95 \%$ confidence interval (CI) of the difference, 1.57-2.10]. Parallel to stress ratings, average MAP increased by $7.30 \mathrm{mmHg}$ (SD, $4.83 \mathrm{mmHg}$ ) from baseline (mean, $81.22 \pm 5.11 \mathrm{mmHg}$ ) to the incongruent condition (mean, $88.52 \pm 6.76 \mathrm{mmHg}$; $95 \%$ CI of the increase, $5.56-9.04 \mathrm{mmHg}$ ).

Men and women did not differ in stress ratings of the congruent (men, mean, $0.83 \pm 0.84$; women, mean, $0.80 \pm 0.62$ ) or incongruent (men, mean, $2.33 \pm 0.89$; women, mean, $2.85 \pm$ 0.67 ) conditions; nor did they differ in their MAP change (men mean $\Delta$ MAP, $8.22 \pm 6.68 \mathrm{mmHg}$; women mean $\Delta \mathrm{MAP}$, $6.75 \pm$ $3.37 \mathrm{mmHg} ; p$ values $>0.05$ by $t$ tests). Given these null findings, remaining results are presented for men and women combined. For consistency, however, sex was retained as an a priori covariate of no interest in all SPM analyses, as detailed in Materials and Methods.

MAP reactivity and amygdala activation As expected, greater MAP reactivity varied with greater amygdala activation to the incongruent condition across individuals (Fig. 2). Notably, MAP reactivity varied with activation in the dorsal portion of the amygdala, extending into the sublenticular area. In humans, the dorsal amygdala encompasses the central nucleus and is thought to support coordinated visceromotor and behavioral arousal processes (Kapp et al., 1992; Whalen, 1998; Somerville et al., 2004).

\section{MAP reactivity and activation in} putative executive control areas In addition to evoking blood pressure reactivity, the incongruent condition of the Stroop task likely engaged several executive control processes, including working memory, conflict and error monitoring, and response selection and execution. Consistent with this notion, a whole-brain analysis showed that the incongruent condition increased BOLD activation in areas implicated in executivecontrol. These areas, referred to recently as components of a "task activation ensemble" (Seeley et al., 2007), included the medial dorsal and mid-anterior cingulate cortex, presupplementary motor area, insula, thalamus, and cerebellum $\left(p_{\mathrm{FWE}}\right.$ values $\left.<0.05\right)$ (supplemental Fig. 1, Table 2, available at www.jneurosci.org as supplemental material). In addition to these areas, an ROI analysis revealed increased amygdala activation to the incongruent condition, which met corrected thresholds in the left amygdala $\left(x, y, z\right.$ MNI coordinates: $-18,-3,-12 ; t_{(31)}=3.49, p_{\text {uncorrected }}$ $\left.=0.001, p_{\mathrm{FWE}}=0.04\right)$, but not the right amygdala $(18,3,-18 ;$ $\left.t_{(31)}=2.69, p_{\text {uncorrected }}=0.006, p_{\mathrm{FWE}}=0.12\right)($ supplemental Fig. 2 , available at www.jneurosci.org as supplemental material).

To test whether MAP reactivity varied with activation in areas outside the amygdala that were specifically engaged by the incongruent condition, we inclusively masked MAP regression contrasts with the parametric map reflecting areas identified in the whole-brain incongruent $>$ congruent condition comparison. We found that MAP reactivity did not vary with activation in these regions ( $p_{\text {uncorrected }}<0.005, k=0$ threshold). This may suggest some dissociation between activation in areas presumably supporting executive-control processes from activation associated specifically with individual differences in MAP reactivity.

\section{MAP reactivity and gray matter volume}

Across individuals, greater MAP reactivity varied with lower amygdala gray mater volume (Fig. 3). Note that in supplemental Figure $3 A$ (available at www.jneurosci.org as supplemental material), the amygdala foci where MAP reactivity varied with gray matter volume overlapped with, but were generally more ventral to, the dorsal foci where MAP reactivity varied with BOLD activation. In humans, the ventral amygdala encompasses the basolateral complex, which is thought to support the convergent pro- 
cessing of behaviorally salient stimuli (Kapp et al., 1992; Whalen, 1998; Somerville et al., 2004). These anatomical comparisons, however, should be cautiously viewed in light of the different spatial resolutions of the BOLD and VBM data.

To test whether MAP reactivity varied with gray matter volume in areas outside the amygdala, we executed an exploratory whole-brain regression analysis that controlled for sex and total gray matter volume. This regression showed that greater MAP reactivity varied with lower gray matter volume in two areas of the medial temporal lobe that extended from the amygdala into the hippocampus and parahippocampal gyrus (supplemental Fig. 4, Table 3, available at www.jneurosci.org as supplemental material). Moreover, as shown in Figure 4, $A$ and $B$, greater MAP reactivity varied with lower gray matter volume in two areas of the medial prefrontal cortex. These areas encompassed Brodmann area (BA) 32 of rostral perigenual anterior cingulate cortex (pACC), extending into BA 10 of the medial prefrontal cortex (supplemental Fig. 4, Table 3, available at www.jneurosci.org as supplemental material).

\section{MAP reactivity and perigenual cingulate activation}

It is noteworthy that previous imaging studies document consistent associations between pACC and medial prefrontal activation and indicators of stressor-evoked cardiac-autonomic and blood pressure reactivity (Critchley, 2005; Gianaros et al., 2005). Also noteworthy is that longstanding animal evidence has established that the pACC and medial prefrontal cortex are involved in autonomic-cardiovascular regulation (Verbene and Owens, 1998). Accordingly, we executed ROI analyses testing whether MAP reactivity varied with pACC activation across individuals. For these analyses, we created anatomically delimited ROIs of the pACC clusters identified in the VBM regressions (supplemental Fig. 4, available at www.jneurosci.org as supplemental material). We maintained ROI false positive detection rates at $p<$ 0.05 within the left $\left(2997 \mathrm{~mm}^{3}\right)$ and right $\left(2565 \mathrm{~mm}^{3}\right)$ pACC volumes by using height and extent thresholds determined by Monte Carlo simulation, as in other ROI analyses (Fig. 4). Consistent with previous studies, greater MAP reactivity varied with greater pACC activation (Fig. 4C,D).

Gray matter volume dependency of MAP reactivity and BOLD activation relationships

Given the proximity between foci of the amygdala and pACC where MAP reactivity varied with activation and gray matter volume, we tested whether the relationships between MAP reactivity and amyg-
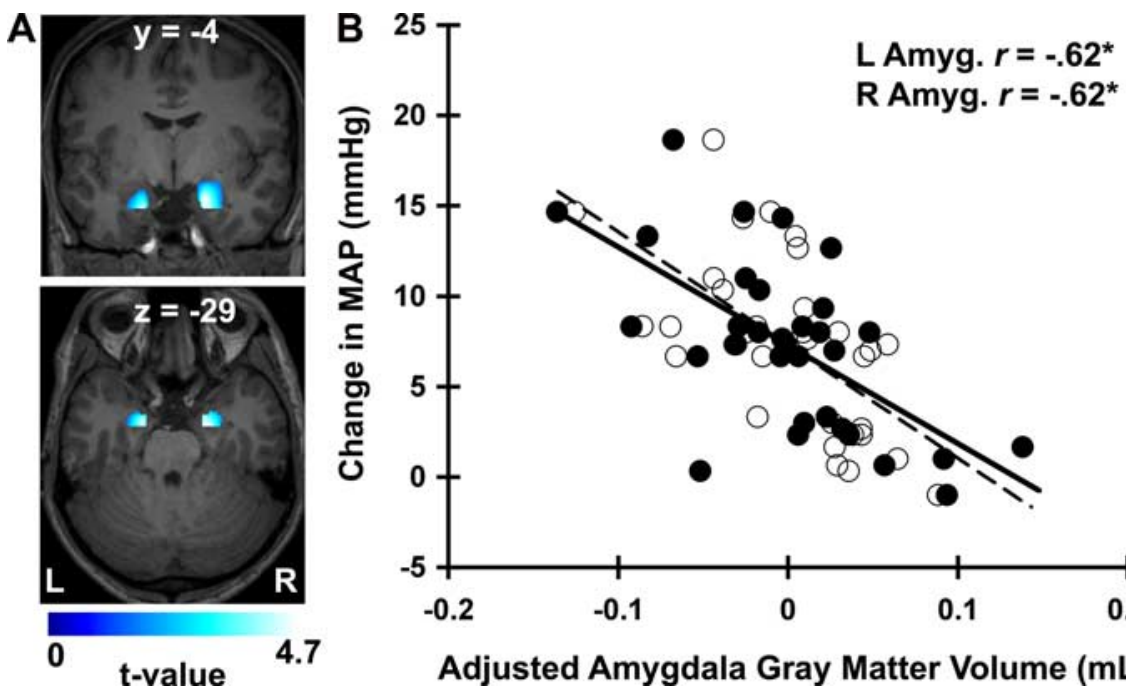

Figure 3. Greater MAP reactivity varied with lower amygdala gray matter volume. $A$, Statistical parametric maps from an $R O$ regression analysis identifying left $\left(x, y, z\right.$ MNI coordinates: $-19,-4,-24 ; t_{(28)}=4.32, p_{\text {uncorrected }}<0.001, p_{\mathrm{FWE}}=0.02, k=$ $470)$ and right $\left(21,-4,-24 ; t_{(28)}=4.29, p_{\text {uncorrected }}<0.001, p_{\mathrm{FWE}}=0.02, k=226\right)$ amygdala clusters where MAP reactivity varied with gray matter volume after covariate control for sex and total gray matter volume. $\boldsymbol{B}$, MAP reactivity is shown as a function of mean-centered and sex- and total gray matter-adjusted volume values extracted from the peak voxels of the left $\left(L_{;}\right.$ open circles, dashed line) and right ( $R$; closed circles, solid line) amygdala clusters in $\boldsymbol{A} .{ }^{*} p<0.01$.
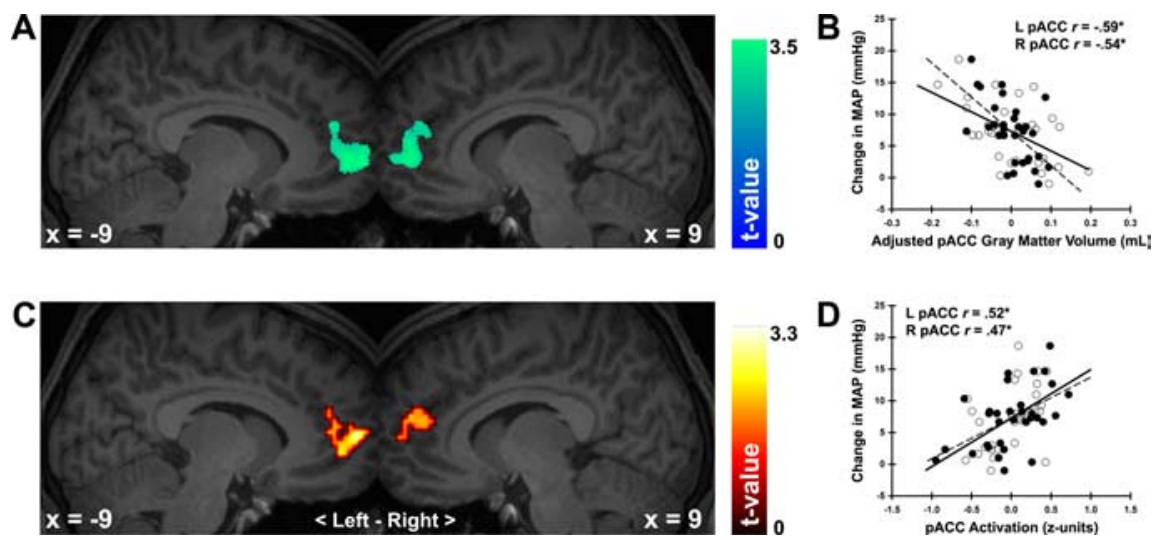

Figure 4. Greater MAP reactivity varied with lower gray matter volume and greater $B O L D$ activation in two areas of the medial prefrontal cortex, which encompassed BA 32 of the pACC and BA 10 of the medial prefrontal cortex. $A$, Statistical parametric maps derived from a whole-brain multiple regression analysis ( $p<0.005, k=750$ threshold) identifying two pACC clusters where MAP reactivity varied with gray matter volume (determined by optimized VBM) after covariate control for sex and total gray matter volume (left pACC $x, y, z$ MNI coordinates: $-4,58,23 ; t_{(28)}=3.96, p_{\text {uncorrected }}<0.001, k=2402$; right pACC: $10,56,4$; $t_{(28)}=4.49, p_{\text {uncorrected }}=0.001, k=1825$ ) (supplemental Fig. 3, Table 3, available at www.jneurosci.org as supplemental material). $\boldsymbol{B}$, MAP reactivity is shown as a function of mean-centered and sex- and total gray matter-adjusted volume values extracted from the peak voxels of the left ( $L$; open circles, dashed line) and right ( $R$; closed circles, solid line) pACC clusters in A.C, Parametric maps from an ROI regression analysis of the VBM-determined pACC clusters identifying areas where greater MAP reactivity varied with greater BOLD activation after covariate control for sex (left pACC: $-9,63,6 ; t_{(29)}=3.32, p_{\text {uncorrected }}=$ $0.001, p_{\mathrm{FWE}}=0.027 ; k=74$; right pACC: $\left.15,60,6 ; t_{(29)}=2.85, p_{\text {uncorrected }}=0.004, p_{\mathrm{FWE}}=0.05, k=45\right) . D$, MAP reactivity is shown as a function of mean-centered and standardized $\mathrm{pACC} B 0 \mathrm{LD}$ activation values extracted from the peak voxels of the left (open circles, dashed line) and right (closed circles, solid line) pACC clusters in $C^{*} p<0.01$.

dala and pACC activation may have depended on gray matter volume. Initial univariate correlations showed that extracted amygdala and pACC gray matter volumes varied inversely with extracted ipsilateral BOLD activation values (left amygdala $r_{(32)}=-0.36, p=$ 0.04 ; right amygdala $r_{(32)}=-0.61, p<0.001$; left pACC $r_{(32)}=$ $-0.37, p=0.04$; right pACC $r_{(32)}=-0.42, p=0.02$ ) (supplemental Figs. $3 B, 5$, Table 4 , available at www.jneurosci.org as supplemental material). These correlations suggested that individuals who expressed lower amygdala and pACC gray matter volumes expressed greater BOLD activation in these areas. 

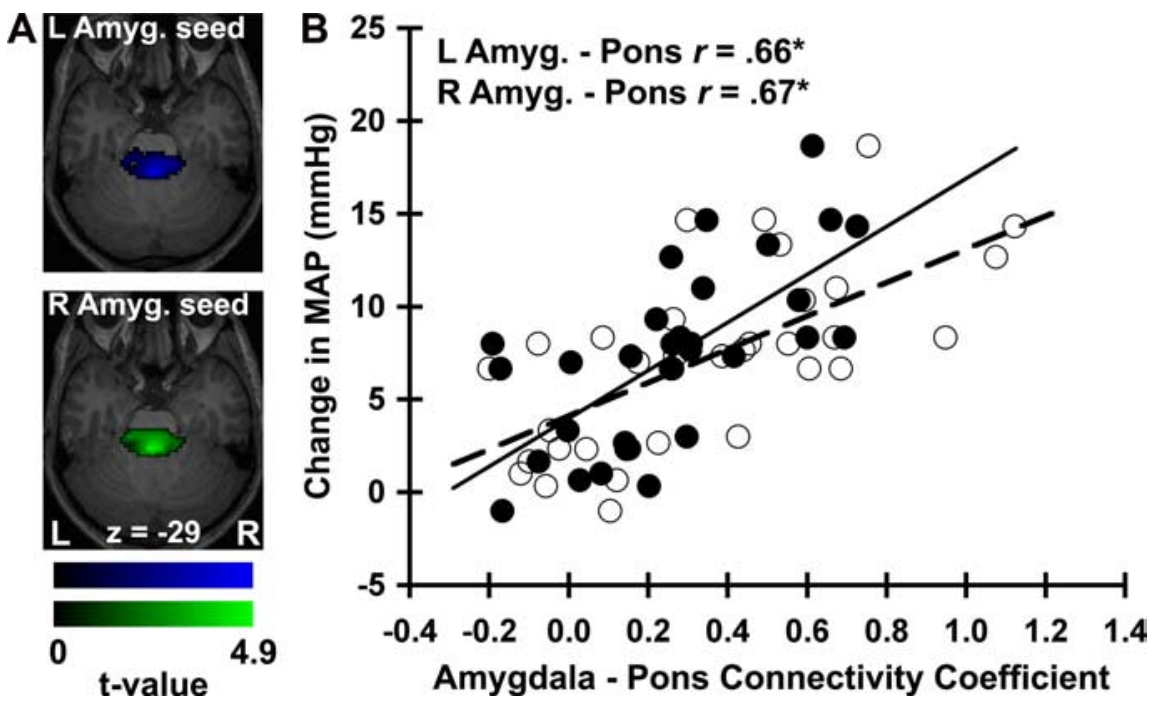

Figure 5. Greater MAP reactivity varied with stronger positive amygdala-pons functional connectivity. $\boldsymbol{A}$, Statistical parametric maps derived from an $\mathrm{ROI}$ regression analysis identifying pons areas where MAP reactivity varied as a function of connectivity with left (top) and right (bottom) amygdala seed regions. Note that BOLD signal seed values from the left ( $x, y, z$ MNI coordinates: $-27,0,-15)$ and right $(27,3,-12)$ amygdala temporally covaried with BOLD activity in overlapping pons areas in association with MAP reactivity (pons coordinates identified from left amygdala seed: $0,-39,-27 ; t_{(29)}=4.69, p_{\text {uncorrected }}=0.001, p_{\mathrm{FWE}}$ $=0.012, k=71 ;$ pons coordinates from right amygdala seed: $-6,-39,-27 ; t_{(29)}=4.86, p_{\text {uncorrected }}=0.001, p_{\mathrm{FWE}}=0.007$, $k=98$ ) (supplemental Fig. 6, available at www.jneurosci.org as supplemental material). $\boldsymbol{B}$, MAP reactivity is plotted as a function of amygdala-pons connectivity coefficients for the left ( $\mathrm{L}$; open circles, dashed line) and right ( $\mathrm{R}$; closed circles, solid line) amygdala seeds. ${ }^{*} p<0.01$.
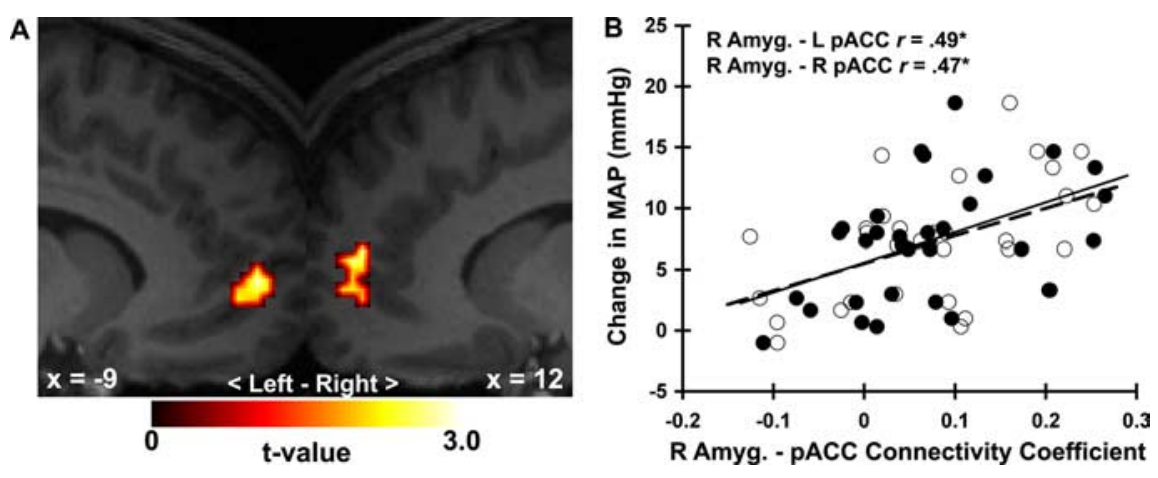

Figure 6. Greater MAP reactivity varied with stronger positive functional connectivity between the right amygdala and pACC. $A$, Statistical parametric maps from ROI regression analyses illustrating areas of the pACC where MAP reactivity varied as a function of connectivity with a right amygdala seed ( $5 \mathrm{~mm}$ sphere surrounding $x, y, z \mathrm{MNI}$ coordinates: $27,3,-12$; left pACC coordinates: $-9,-60,3 ; t_{(29)}=2.97, p_{\mathrm{FWE}}=0.08, p_{\text {uncorrected }}=0.003, k=15$; right pACC coordinates: $12,57,12 ; t_{(29)}=$ $2.87, p_{\mathrm{FWE}}=0.07, p_{\text {uncorrected }}=0.004, k=16$ ) (supplemental Fig. 7, available at www.jneurosci.org as supplemental material). $B$, MAP reactivity is shown as a function of right amygdala-left $p A C C$ ( $L$; open circles, dashed line) and right amygdala-right pACC (R; closed circles, solid line) connectivity coefficients. ${ }^{*} p<0.01$.

Partial correlation analyses next demonstrated that controlling for gray matter volume rendered the relationships between MAP reactivity and amygdala activation statistically nonsignificant (left amygdala, partial correlation coefficient, $\operatorname{pr}_{(28)}=0.18$, $p=0.35$; right amygdala $\left.p r_{(28)}=0.28, p=0.13\right)$. Conversely, the relationships between MAP reactivity and amygdala gray matter volume were unaltered after controlling for amygdala activation (left amygdala $p r_{(28)}=-0.51, p=0.004$; right amygdala $p r=$ $-0.41, p=0.03)$. These correlations paralleled those for the pACC: controlling for gray matter volume attenuated the relationships between MAP reactivity and pACC activation to nonsignificance (left pACC $p r_{(28)}=0.28, p=0.13$; right pACC $p r_{(28)}$ $=0.30, p=0.11)$. In contrast, the relationships between MAP reactivity and pACC gray matter volume persisted after control for pACC activation (left pACC $p r_{(28)}=$ $-0.50, p=0.005$; right pACC $\operatorname{pr}_{(28)}=$ $-0.36, p=0.05)$.

Additional control for sex and total gray matter volume affected neither the direction nor significance of any of these correlations. These findings suggest that the relationships between MAP reactivity and amygdala and pACC activation may depend on gray matter volume.

\section{MAP reactivity as a function of amygdala-pons and amygdala-pACC connectivity Pons}

To test whether the pons represents a component of a putative preautonomic cardiovascular control circuit that relates individual differences in amygdala activation to MAP reactivity, we executed an ROI regression analysis that examined MAP reactivity as a function of amygdala-pons connectivity. The regression showed that greater MAP reactivity varied with stronger positive functional connectivity between the amygdala and pons (Fig. 5, supplemental Fig. 6, available at www.jneurosci.org as supplemental material).

Of note, a supplementary ROI analysis revealed no association between MAP reactivity and pons activation to the incongruent condition ( $p_{\text {uncorrected }}<0.005, k=0$ threshold). Another ROI analysis of the VBM data revealed no association between MAP reactivity and pons gray matter volume ( $p_{\text {uncorrected }}<0.005, k=0$ threshold). These findings suggest a relative specificity for amygdala-pons connectivity in association with MAP reactivity.

\section{pACC}

Given the established anatomical and functional connectivity between the amygdala and pACC (Amaral and Price, 1984; McDonald, 1998; Pezawas et al., 2005; Vogt, 2005), in addition to previous work noted above documenting an association between pACC activation and autonomiccardiovascular reactivity, we executed ROI analyses to test whether MAP reactivity varied with amygdala-pACC functional connectivity. For the analyses, we used the same anatomically delimited ROI masks of the pACC clusters reported above. We maintained false positive detection rates at $p<0.05$ within the ROI volumes by using height and extent thresholds determined by Monte Carlo simulation (Fig. 6). We found that greater MAP reactivity varied with stronger positive connectivity between the right amygdala and bilateral pACC (Fig. 6), particularly after removing an outlier with amygdala-pACC connectivity values $>3 \mathrm{SD}$ above the mean (supplemental Fig. 7, available at www.jneurosci.org as supplemental material). The relationships between MAP reactivity and left amygdala connectivity with the left and right pACC, however, did not reach significance at corrected and uncorrected thresholds. 
Task-related amygdala-connectivity with corticolimbic areas To identify areas exhibiting functional connectivity with the amygdala during the Stroop task, we executed whole-brain analyses for the left $(-27,0,-15)$ and right $(27,3,-12)$ amygdala seed regions where greater MAP reactivity varied with greater activation. These analyses identified corticolimbic areas known to express anatomical and functional connectivity with the amygdala (Amaral and Price, 1984; McDonald, 1998; Ghashghaei and Barbas, 2002; Pezawas et al., 2005; Seeley et al., 2007): the pACC, ventrolateral orbitofrontal cortex bordering the anterior insula, hippocampus, parahippocampal gyrus, caudate, inferior and middle temporal gyri, precentral and postcentral gyri, occipital cortex, and cerebellum $\left(p_{\mathrm{FWE}}\right.$ values $<0.001, k$ values $>15$ voxels) (supplemental Fig. 8, Table 5, available at www. jneurosci.org as supplemental material). Although identified post hoc, these areas may represent components of an amygdalaanchored network supporting stressor-processing in the behavioral context of our blood pressure reactivity task (see Discussion).

Supplementary analyses of the insula and posterior cingulate Our primary analytic focus centered on the amygdala. For completeness of reporting, however, we examined two other areas suggested by previous imaging studies to be involved in autonomic-cardiovascular control and reactivity: the insula and posterior cingulate (Critchley, 2005; Dalton et al., 2005; Gianaros et al., 2005; Wong et al., 2007). For these analyses, we created bilateral masks of each area with the WFU Pick Atlas, and we used Monte Carlo-determined thresholds $(p<0.005, k$ values $>8)$ to maintain ROI false-positive detection rates at $p<0.05$. Consistent with previous findings, greater MAP reactivity varied with greater activation in the anterior insula (left, $-24,15,-21, t_{(29)}=$ $3.35, p_{\text {uncorrected }}=0.001, p_{\mathrm{FWE}}=0.17, r=0.52, p=0.002$; right, $27,21,-15, t_{(29)}=3.57, p_{\text {uncorrected }}=0.001, p_{\mathrm{FWE}}=0.11, r=$ $0.55, p=0.001)$ and posterior cingulate cortex (left, $-6,-36,27$, $t_{(29)}=3.16, p_{\text {uncorrected }}=0.002, p_{\mathrm{FWE}}=0.07, r=0.50, p=0.004$; right, $6,-36,30, t_{(29)}=3.43, p_{\text {uncorrected }}=0.001, p_{\mathrm{FWE}}=0.04$, $r=0.53, p=0.002$ ). MAP reactivity, however, did not vary with gray matter volume in these areas or with the functional connectivity between these areas and the amygdala. Hence, insula and posterior cingulate activation could relate to individual differences in MAP reactivity through functional pathways that may not necessarily involve the amygdala (see Discussion).

\section{Discussion}

This study provides four lines of evidence that the amygdala could play a role in mediating individual differences in stressorevoked blood pressure reactivity, which are linked to incident cardiovascular pathophysiology and clinical risk. First, individuals exhibiting greater stressor-evoked MAP reactivity showed concurrently greater amygdala activation compared with their less reactive counterparts. Second, individuals exhibiting greater MAP reactivity had a lower amygdala gray matter volume, which itself predicted greater amygdala activation. Third, individuals exhibiting greater MAP reactivity showed stronger positive amygdala-pons functional connectivity. Fourth, individuals exhibiting greater MAP reactivity showed greater activation and a correspondingly lower gray matter volume in the pACC, as well as stronger positive amygdala-pACC functional connectivity.

\section{Possible pathways linking the amygdala with blood pressure reactivity}

As a functionally integrated cell complex, the amygdala is thought to process behaviorally salient stimuli, particularly threatening, ambiguous, unpredictable, and stressful stimuli (Whalen, 1998; LeDoux, 2000; Sah et al., 2003; Zald, 2003). To provide hemodynamic support for adaptive responses to such stimuli, the amygdala can regulate changes in blood pressure through multiple neuroanatomical pathways (LeDoux et al., 1982; Reis and LeDoux, 1987; Dampney, 1994; Saha, 2005). A direct pathway involves amygdala control over the baroreflex, a homeostatic mechanism that constrains arterial pressure around a regulatory set-point (Dampney, 1994; Berntson et al., 1998; Saha, 2005). Through coordinated changes in autonomic nervous system activity, the baroreflex constrains beat-by-beat blood pressure by adjusting heart rate, cardiac output, and vascular resistance to support contextually appropriate behavior. As a negativefeedback loop, the baroreflex relies on afferent projections from cardiopulmonary mechanoreceptors and chemoreceptors that signal changes in blood pressure to the nucleus of the solitary tract (NTS). The NTS in turn activates vagal parasympathetic nuclei in the medulla (nucleus ambiguous, dorsal motor nucleus), and via signaling with the caudal ventrolateral medulla, inhibits presympathetic nuclei in the rostral ventrolateral medulla and cell groups in the spinal intermediolateral column. Critically, during aversive or stressful behavioral states, the central nucleus of the amygdala can gate the baroreflex via inhibitory projections to the NTS and via excitatory projections to the rostral ventrolateral medulla. These projections, which are relayed through brainstem areas including the pons, effectively suppress parasympathetic cardiac control and redirect sympathetic vascular outflow, allowing blood pressure to exceed a regulated setpoint (Dampney, 1994; Berntson et al., 1998; Saha, 2005).

Based on these pathways, Berntson and colleagues (1998) speculated that individual differences in amygdala activation to behaviorally salient stimuli could mediate corresponding differences in autonomic-cardiovascular reactivity. Our finding that greater MAP reactivity varied with greater dorsal amygdala activation agrees with this speculation. This finding also agrees with previous evidence that amygdala activation to behaviorally salient stimuli covaries with changes in myocardial contractility (Dalton et al., 2005), arterial pressure, and heart rate (Critchley et al., 2000, 2005). This finding also complements evidence (Urry et al., 2006) that individuals who express greater amygdala activation to emotionally salient stimuli show a flatter diurnal secretion of cortisol, a form of dysregulated neuroendocrine activity associated with risk for atherosclerosis (Matthews et al., 2006a). Here, however, we did not assess multiple autonomic, neuroendocrine, or cardiovascular measures, constraining our conclusions to blood pressure reactivity. Nevertheless, in view of existing evidence, our findings support the notion that dysregulated amygdala activity may influence human cardiovascular risk by impacting several autonomic, neuroendocrine, and cardiovascular control mechanisms.

Extending the BOLD activation findings, functional connectivity findings suggested that the pons may be a preautonomic area that links amygdala activity with MAP reactivity. The amygdala expresses reciprocal connections with pontine cell groups critical for cardiovascular control (Hopkins and Holstege, 1978; Miller et al., 1991; Dampney, 1994). Furthermore, the pons relays afferent cardiovascular information to higher levels of the neuroaxis, including the amygdala (Dampney, 1994). In speculation, individual differences in blood pressure reactivity could result from differential signaling between the amygdala and preautonomic areas such as the pons. In point, stronger efferent amygdala-preautonomic signaling could reflect stronger descending commands for blood pressure rises. In parallel, stronger 
afferent preautonomic-amygdala signaling could reflect stronger ascending negative-feedback to the amygdala that functionally curtails excessive blood pressure rises. The latter possibility may account for the finding that individuals showing more negative amygdala-pons connectivity showed lesser MAP reactivity. Our connectivity data, however, do not distinguish efferent from afferent signaling patterns linked to blood pressure changes, which were measured on a minute-by-minute basis. Moreover, we did not examine BOLD activity in subpontine preautonomic areas (e.g., medulla). Future studies should thus measure more temporally precise BOLD signal changes in relation to beat-by-beat blood pressure and employ effective connectivity procedures (Friston, 1994) to parse efferent from afferent neural signaling patterns in the amygdala and preautonomic areas putatively involved in cardiovascular control.

\section{Gray matter volume and possible vulnerability for dysregulated blood pressure reactivity}

Optimized VBM findings showed that greater MAP reactivity varied with lower amygdala and pACC gray matter volumes. Furthermore, the relationships between BOLD activation and MAP reactivity statistically depended on gray matter volume in these areas. These findings highlight the importance of accounting for normative variation in brain morphology in examining the relationships between blood pressure reactivity and associated neural activation patterns. However, our cross-sectional design and the nonspecific nature of VBM data regarding cell structure and density do not permit inferences about the causal or cellular mechanisms linking gray matter volume with BOLD activation and MAP reactivity. One possibility is that genetic influences on corticolimbic development could modify activation to behaviorally salient stimuli. This is suggested by work demonstrating that a functional polymorphism of the serotonin transporter gene, which alters serotonin signaling linked to neurodevelopmental plasticity, is associated with reduced amygdala and pACC volumes, greater amygdala reactivity, and dysregulated amygdalapACC coupling (Pezawas et al., 2005). In view of such genetic contributions, future longitudinal studies should assay genomic markers and test whether genetically driven variation in corticolimbic development may underpin vulnerability for dysregulated neural activation and connectivity in association with blood pressure reactivity and possible cardiovascular risk.

\section{Amygdala-cingulate interactions and blood pressure reactivity}

Greater MAP reactivity varied with greater pACC activation and with more positive amygdala-pACC functional connectivity. The amygdala and pACC are densely networked, and both areas are considered as components of a corticolimbic circuit that orchestrates adaptive neurobehavioral and visceromotor stress responses (Devinsky et al., 1995; Bush et al., 2000; Paus, 2001; Vogt, 2005). Furthermore, evidence from fear-conditioning and emotion-regulation literatures suggests that subdivisions of the anterior cingulate, particularly in the perigenual area, may regulate amygdala activity (Ochsner and Gross, 2005; Etkin et al., 2006; Quirk and Beer, 2006). Hence, if regulatory amygdalapACC signaling modulates visceromotor reactivity to behaviorally salient stimuli, then the differential coupling between these areas may partly influence individual differences in stressorevoked blood pressure reactivity. Consistent with this notion, individuals expressing more negative amygdala-pACC connectivity expressed lesser MAP reactivity. To our knowledge, however, no published studies have examined amygdala-pACC con- nectivity in association with blood pressure reactivity. Therefore, our findings, like those relating amygdala-pons connectivity with MAP reactivity, should be provisionally interpreted until future studies replicate our observations and explicate the putatively regulatory (e.g., bottom up vs top down) nature of amygdalapACC signaling in association with cardiovascular control.

\section{Limitations and future directions}

To elicit blood pressure reactivity, we used a Stroop stressor that differs from paradigms often used to engage the amygdala (e.g., picture viewing, emotion regulation). We used this stressor because it is used in epidemiological studies of cardiovascular risk, providing direct relevance to understanding amygdala-based processes possibly underlying cardiovascular risk. The Stroop stressor likely elicits blood pressure reactivity because it engages performance-titrated conflict resolution processes, involves trial unpredictably, and entails negative performance feedback. This task paradigm could thus be viewed as a "psychological stressor" that does not necessarily elicit a discrete emotional state. Therefore, future studies should determine whether individual differences in amygdala activation to more explicit emotionally laden paradigms (e.g., fear conditioning) predict blood pressure or other cardiovascular reactions associated with cardiovascular risk (cf., Dalton et al., 2005).

We also did not account for individual differences in executive function or cognitive ability, which could affect Stroop task performance. Task performance, however, was adaptively maintained at comparable levels across individuals. Also, an interesting finding was that MAP reactivity did not vary with activation in prefrontal, dorsal anterior cingulate, insular, thalamic, and cerebellar areas specifically engaged by the incongruent condition, areas which presumably support executive-control processes. This finding may be interpreted by a conceptual perspective derived from the recent identification of two "intrinsic connectivity networks" that function in parallel under resting and active behavioral states (Seeley et al., 2007). One network, termed a dorsal neocortical system is thought to support executive-control processes. The other, termed a paralimbic salience system, includes the amygdala and is thought to process behaviorally salient stimuli and regulate changes in visceromotor activity. Although identified under different conditions than those reported by Seeley et al. (2007), it is noteworthy that the amygdala expressed functional connectivity with many areas of the paralimbic salience system during the Stroop task. Given these results, an interesting future research direction would be to determine whether individual differences in these connectivity patterns could functionally link autonomic-cardiovascular reactivity with dispositional characteristics (e.g., negative emotionality) or subjective states (e.g., anxiety) previously associated with amygdala activation (Etkin et al., 2004) and connectivity in the paralimbic salience system (Seeley et al., 2007).

Finally, our ongoing work is attempting to delineate the pathways that link blood pressure reactivity with activation in the insula and posterior cingulate. Notably, neither area showed volumetric changes or differential amygdala-connectivity in association with MAP reactivity. Although densely networked with the amygdala, the insula can regulate cardiovascular function through autonomic mechanisms that do not entail an obligatory amygdala pathway (Yasui et al., 1991; Cechetto, 1994; Verbene and Owens, 1998). Furthermore, the posterior cingulate is a functionally heterogeneous area that is indirectly networked with the amygdala and not extensively networked with autonomiccardiovascular control areas (Mayberg, 1997; Vogt et al., 2006; 
Stein et al., 2007). As speculated previously, posterior cingulate activity likely relates to indicators of autonomic-cardiovascular control via pathways relayed through perigenual cingulate areas more proximally involved in autonomic-cardiovascular regulation (Gianaros et al., 2005; O'Connor et al., 2007).

In summary, our findings suggest a role for the amygdala and networked corticolimbic and preautonomic areas in mediating individual differences in a parameter of cardiovascular risk: stressor-evoked blood pressure reactivity. Individuals who exhibit 'exaggerated' stressor-evoked blood pressure reactions are at risk for developing precursors to cardiovascular disease. As one component of a distributed corticolimbic circuit, the amygdala has long been implicated in sensitivity and resiliency to stress, particularly in the context of depressive and anxiety disorders (Mayberg, 1997; Drevets, 2000; Greicius et al., 2007). Importantly, these stress-related disorders confer comorbid risk for cardiovascular disease and involve dysregulated control over autonomic, cardiovascular, and neuroendocrine activity (Hemingway and Marmot, 1999; Rozanski et al., 1999). A question with clinical relevance is whether amygdala activation, morphology, or coupling with corticolimbic and preautonomic areas predict biomarkers or endpoints of cardiovascular disease, especially among those with high stress-related psychiatric and cardiovascular comorbidity.

\section{References}

Amaral DG, Price JL (1984) Amygdalo-cortical projections in the monkey (Macaca fascicularis). J Comp Neurol 230:465-496.

Ashburner J, Friston KJ (2000) Voxel-based morphometry: the methods. NeuroImage 11:805-821.

Baklavadzhyan OG, Pogosyan NL, Arshakyan AV, Darbinyan AG, Khachatryan AV, Nikogosyan TG (2000) Studies of the role of the central nucleus of the amygdala in controlling cardiovascular functions. Neurosci Behav Phys 30:231-236.

Berntson GG, Sarter M, Cacioppo JT (1998) Anxiety and cardiovascular reactivity: the basal forebrain cholinergic link. Behav Brain Res 94:225-248.

Brett M, Anton J, Valabregue R, Poline J (2002) Region of interest analysis using an SPM toolbox. NeuroImage 16:S497.

Bush G, Luu P, Posner MI (2000) Cognitive and emotional influences in anterior cingulate cortex. Trends Cogn Sci 4:215-222.

Cannon WB (1928) The mechanism of emotional disturbance of bodily functions. N Engl J Med 198:877-884.

Cechetto DF (1994) Identification of a cortical site for stress-induced cardiovascular dysfunction. Integr Physiol Behav Sci 29:362-373.

Critchley HD (2005) Neural mechanisms of autonomic, affective, and cognitive integration. J Comp Neurol 493:154-166.

Critchley HD, Corfield DR, Chandler MP, Mathias CJ, Dolan RJ (2000) Cerebral correlates of autonomic cardiovascular arousal: a functional neuroimaging investigation in humans. J Physiol (Lond) 523:259-270.

Critchley HD, Rotshtein P, Nagai Y, O’Doherty J, Mathias CJ, Dolan RJ (2005) Activity in the human brain predicting differential heart rate responses to emotional facial expressions. NeuroImage 24:751-762.

Cuadra MB, Cammoun L, Butz T, Cuisenaire O, Thiran JP (2005) Comparison and validation of tissue modelization and statistical classification methods in T1-weighted MR brain images. IEEE Trans Med Imaging 24:1548-1565.

Dalton KM, Kalin NH, Grist TM, Davidson RJ (2005) Neural-cardiac coupling in threat-evoked anxiety. J Cogn Neurosci 17:969-980.

Dampney RA (1994) Functional organization of central pathways regulating the cardiovascular system. Physiol Rev 74:323-364.

Davis M, Whalen PJ (2001) The amygdala: vigilance and emotion. Mol Psychiatry $6: 13-34$

Devinsky O, Morrell MJ, Vogt BA (1995) Contributions of anterior cingulate cortex to behaviour. Brain 118:279-306

Drevets WC (2000) Functional anatomical abnormalities in limbic and prefrontal cortical structures in major depression. Prog Brain Res 126:413-431.

Etkin A, Klemenhagen KC, Dudman JT, Rogan MT, Hen R, Kandel ER,
Hirsch J (2004) Individual differences in trait anxiety predict the response of the basolateral amygdala to unconsciously processed fearful faces. Neuron 44:1043-1055.

Etkin A, Egner T, Peraza DM, Kandel ER, Hirsch J (2006) Resolving emotional conflict: a role for the rostral anterior cingulate cortex in modulating activity in the amygdala. Neuron 51:871-882.

Forman SD, Cohen JD, Fitzgerald M, Eddy WF, Mintun MA, Noll DC (1995) Improved assessment of significant activation in functional magnetic resonance imaging (fMRI): use of a cluster-size threshold. Magn Reson Med 33:636-647.

Friston KJ (1994) Functional and effective connectivity in neuroimaging: a synthesis. Hum Brain Mapp 2:56-78.

Friston KJ, Holmes AP, Worsley KJ, Poline JP, Frith CD, Frackowiak RSJ (1995) Statistical parametric maps in functional imaging: a general approach. Hum Brain Mapp 2:189-210.

Friston KJ, Holmes A, Poline JB, Price CJ, Frith CD (1996) Detecting activations in PET and fMRI: levels of inference and power. NeuroImage 4:223-235.

Fukumori R, Nishigori Y, Goshima Y, Kubo T (2004) Contribution of the medial amygdaloid nucleus to the development of hypertension in spontaneously hypertensive rats. Neurosci Lett 365:128-131.

Galeno TM, Van Hoesen GW, Brody MJ (1984) Central amygdaloid nucleus lesion attenuates exaggerated hemodynamic responses to noise stress in the spontaneously hypertensive rat. Brain Res 291:249-259.

Ghashghaei T, Barbas H (2002) Pathways for emotion: interactions of prefrontal and anterior temporal pathways in the amygdala of rhesus monkey. Neurosci 115:1261-1279.

Gianaros PJ, Derbyshire SW, May JC, Siegle GJ, Gamalo MA, Jennings JR (2005) Anterior cingulate activity correlates with blood pressure during stress. Psychophysiology 42:627-635.

Good CD, Johnsrude IS, Ashburner J, Henson RN, Friston KJ, Frackowiak RS (2001) A voxel-based morphometric study of ageing in 465 normal adult human brains. NeuroImage 14:21-36.

Greicius MD, Krasnow B, Reiss AL, Menon V (2003) Functional connectivity in the resting brain: a network analysis of the default mode hypothesis. Proc Natl Acad Sci USA 100:253-258.

Greicius MD, Flores BH, Menon V, Glover GH, Solvason HB, Kenna H, Reiss AL, Schatzberg AF (2007) Resting-state functional connectivity in major depression: abnormally increased contributions from subgenual cingulate cortex and thalamus. Biol Psychiatry 62:429-437.

Hemingway H, Marmot M (1999) Psychosocial factors in the aetiology and prognosis of coronary heart disease: systematic review of prospective cohort studies. BMJ 318:1460-1467.

Hopkins DA, Holstege G (1978) Amygdaloid projections to the mesencephalon, pons and medulla oblongata in the cat. Exp Brain Res 32:529-547.

Jennings J, Kamarck T, Everson-Rose S, Kaplan G, Manuck S, Salonen J (2004) Exaggerated blood pressure responses during mental stress are prospectively related to enhanced carotid atherosclerosis in middle-aged Finnish men. Circulation 110:2198-2203.

Kamarck TW, Lovallo WR (2003) Cardiovascular reactivity to psychological challenge: conceptual and measurement considerations. Psychosom Med 65:9-21.

Kapp B, Whalen P, Pascoe J, Supple W (1992) Amygdaloid contributions to conditioned arousal and sensory information processing. In: The amygdala: neurobiological aspects of emotion, memory and mental dysfunction (Aggleton J, ed), p 229-254. New York: Wiley.

Krantz DS, Manuck SB (1984) Acute psychophysiologic reactivity and risk of cardiovascular disease: a review and methodologic critique. Psychol Bull 96:435-464.

LeDoux JE (2000) Emotion circuits in the brain. Annu Rev Neurosci 23:155-184.

LeDoux JE, Del Bo A, Tucker LW, Harshfield G, Talman WT, Reis DJ (1982) Hierarchic organization of blood pressure responses during the expression of natural behaviors in rat: mediation by sympathetic nerves. Exp Neurol 78:121-133.

Maldjian JA, Laurienti PJ, Kraft RA, Burdette JH (2003) An automated method for neuroanatomic and cytoarchitectonic atlas-based interrogation of fMRI data sets. NeuroImage 19:1233-1239.

Matthews K, Schwartz J, Cohen S, Seeman T (2006a) Diurnal cortisol decline is related to coronary calcification: CARDIA study. Psychosom Med 68:657-661.

Matthews KA, Zhu S, Tucker DC, Whooley MA (2006b) Blood pressure 
reactivity to psychological stress and coronary calcification in the Coronary Artery Risk Development in Young Adults Study. Hypertension 47:391-395.

Mayberg HS (1997) Limbic-cortical dysregulation: a proposed model of depression. J Neuropsychiatry Clin Neurosci 9:471-481.

McDonald AJ (1998) Cortical pathways to the mammalian amygdala. Prog Neurobiol 55:257-332.

Miller Jr FJ, Marcus ML, Brody MJ, Gutterman DD (1991) Activation in the region of parabrachial nucleus elicits neurogenically mediated coronary vasoconstriction. Am J Physiol (Lond) 261:H1585-H1596.

O'Connor MF, Gundel H, McRae K, Lane RD (2007) Baseline vagal tone predicts BOLD response during elicitation of grief. Neuropsychopharmacology 32:2184-2189.

Ochsner KN, Gross JJ (2005) The cognitive control of emotion. Trends Cogn Sci 9:242-249.

Öngür D, Price J (2000) The organization of networks within the orbital and medial prefrontal cortex of rats, monkeys, and humans. Cereb Cortex 10:206-219.

Paus T (2001) Primate anterior cingulate cortex: where motor control, drive, and cognition interface. Nat Neurosci Rev 2:417-424.

Pezawas L, Meyer-Lindenberg A, Drabant EM, Verchinski BA, Munoz KE, Kolachana BS, Egan MF, Mattay VS, Hariri AR, Weinberger DR (2005) 5-HTTLPR polymorphism impacts human cingulate-amygdala interactions: a genetic susceptibility mechanism for depression. Nat Neurosci $8: 828-834$.

Quirk GJ, Beer JS (2006) Prefrontal involvement in the regulation of emotion: convergence of rat and human studies. Curr Opin Neurobiol $16: 723-727$.

Reis DJ, LeDoux JE (1987) Some central neural mechanisms governing resting and behaviorally coupled control of blood pressure. Circulation 76:12-9.

Rozanski A, Blumenthal JA, Kaplan J (1999) Impact of psychological factors on the pathogenesis of cardiovascular disease and implications for therapy. Circulation 99:2192-2217.

Sah P, Faber ES, Lopez De Armentia M, Power J (2003) The amygdaloid complex: anatomy and physiology. Physiol Rev 83:803-834.

Saha S (2005) Role of the central nucleus of the amygdala in the control of blood pressure: descending pathways to medullary cardiovascular nuclei. Clin Exp Pharmacol Physiol 32:450-456.

Sanders BJ, Wirtz-Nole C, DeFord SM, Erling BF (1994) Central amygda- loid lesions attenuate cardiovascular responses to acute stress in rats with borderline hypertension. Physiol Behav 56:709-713.

Seeley WW, Menon V, Schatzberg AF, Keller J, Glover GH, Kenna H, Reiss AL, Greicius MD (2007) Dissociable intrinsic connectivity networks for salience processing and executive control. J Neurosci 27:2349-2356.

Somerville LH, Kim H, Johnstone T, Alexander AL, Whalen PJ (2004) Human amygdala responses during presentation of happy and neutral faces: correlations with state anxiety. Biol Psychiatry 55:897-903.

Stein JL, Wiedholz LM, Bassett DS, Weinberger DR, Zink CF, Mattay VS, Meyer-Lindenberg A (2007) A validated network of effective amygdala connectivity. NeuroImage 36:736-745.

Treiber FA, Kamarck T, Schneiderman N, Sheffield D, Kapuku G, Taylor T (2003) Cardiovascular reactivity and development of preclinical and clinical disease states. Psychosom Med 65:46-62.

Urry HL, van Reekum CM, Johnstone T, Kalin NH, Thurow ME, Schaefer HS, Jackson CA, Frye CJ, Greischar LL, Alexander AL, Davidson RJ (2006) Amygdala and ventromedial prefrontal cortex are inversely coupled during regulation of negative affect and predict the diurnal pattern of cortisol secretion among older adults. J Neurosci 26:4415-4425.

Verbene A, Owens N (1998) Cortical modulation of the cardiovascular system. Prog Neurobiol 54:149-168.

Vogt BA (2005) Pain and emotion interactions in subregions of the cingulate gyrus. Nat Rev Neurosci 6:533-544.

Vogt BA, Vogt L, Laureys S (2006) Cytology and functionally correlated circuits of human posterior cingulate areas. NeuroImage 29:452-466.

Ward BD (2000) Simultaneous inference for fMRI data. AFNI AlphaSim documentation. Milwaukee, WI: Biophysics Research Institute, Medical College of Wisconsin. Retrieved Jan. 14, 2008 from http://afni.nimh.nih.gov/pub/dist/doc/manual/AlphaSim.pdf.

Whalen P (1998) Fear, vigilance and ambiguity: initial neuroimaging studies of the human amygdala. Curr Dir Psychol Sci 7:177-188.

Wong SW, Masse N, Kimmerly DS, Menon RS, Shoemaker JK (2007) Ventral medial prefrontal cortex and cardiovagal control in conscious humans. NeuroImage 35:698-708.

Yasui Y, Breder CD, Saper CB, Cechetto DF (1991) Autonomic responses and efferent pathways from the insular cortex in the rat. J Comp Neurol 303:355-374.

Zald DH (2003) The human amygdala and the emotional evaluation of sensory stimuli. Brain Res Brain Res Rev 41:88-123. 